\title{
AVANCE GENÉTICO EN 14 CICLOS DE SELECCIÓN RECURRENTE EN LA POBLACIÓN ANDROESTÉRIL PANDOLY.PABG DE TRIGO
}

\author{
GENETIC ADVANCE IN 14 CYCLES OF RECURRENT SELECTION \\ IN THE PANDOLY.PABG MALE STERILE WHEAT POPULATION
}

\author{
Huizar L. Díaz-Ceniceros ${ }^{1}$, Héctor E. Villaseñor-Mir ${ }^{2}$, Ignacio Benítez-Riquelme ${ }^{1}$, \\ Ma. del Carmen Mendoza-Castillo ${ }^{1}$ y René Hortelano-Santa Rosa ${ }^{2}$
}

\begin{abstract}
'Colegio de Postgraduados, Campus Montecillo, Postgrado en Recursos Genéticos y Productividad-Genética, Montecillo, Texcoco, Estado de México, México. ${ }^{2}$ Instituto Nacional de Investigaciones Forestales, Agricolas y Pecuarias. Campo Experimental Valle de México, Coatlinchán, Texcoco, Estado de México, México.
\end{abstract}

*Autor de correspondencia (hevimir3@yahoo.com.mx)

\section{RESUMEN}

El gen Oly es una fuente de androesterilidad dominante que ha permitido conformar diversas poblaciones para realizar selección recurrente en trigo (Triticum aestivum L.), tal es el caso de la población androestéril de amplia base genética denominada PANDOLY.PABG, que ha permitido derivar líneas y generar variedades. Con el objetivo de estimar el avance genético después de 14 ciclos de selección recurrente en dicha población y valorar la eficiencia del gen Oly para formar líneas superiores, se evaluaron 80 líneas $\mathrm{F}_{5}$ tomadas al azar de los ciclos $\mathrm{C} 0, \mathrm{C} 2, \mathrm{C} 4, \mathrm{C} 6, \mathrm{C} 8, \mathrm{C} 10, \mathrm{C} 12$ y $\mathrm{C} 14$ más 10 variedades testigo. Se empleó un diseño alfa-látice $9 \times 10$ con dos repeticiones y dos fechas de siembra durante el ciclo otoño-invierno 2017/2018 en Roque, Guanajuato y Montecillo, Estado de México. Las variables evaluadas fueron días a floración (DF), altura de planta (AP), días a madurez (DM), rendimiento de grano (RG), tasa de llenado de grano (TLLG), índice de cosecha (IC), biomasa (BIO), peso de 1000 semillas (PMS), número de espigas por $\mathrm{m}^{2}$ (EPM2), de granos por $\mathrm{m}^{2}$ (GPM2) y de granos por espiga (GPE). El mayor aumento de RG fue de $1445 \mathrm{~kg}$ $\mathrm{ha}^{-1}$ entre los ciclos $\mathrm{CO}$ y C2. El avance genético promedio en los 14 ciclos fue de $3.58 \%$ (140.6 $\left.\mathrm{kg} \mathrm{ha}^{-1} / \mathrm{ciclo}\right)$. Entre los cambios morfofisiológicos destacan, particularmente en los ciclos avanzados de selección, aumento $(P \leq 0.05)$ en los caracteres TLLG, IC, BIO, PMS, EPM2, GPM2 y GPE, y disminución ( $\mathrm{P} \leq$ $0.05)$ de DF, DM y AP. Las variedades testigo y las líneas derivadas del C14 presentaron $R G$ más altos $(P \leq 0.05)$ y estadísticamente iguales. El gen Oly es un excelente facilitador del mejoramiento genético poblacional en trigo.

Palabras clave: Triticum aestivum, ciclo de selección, esterilidad masculina, técnica MSFRS

\section{SUMMARY}

The Oly gene is a dominant source of male sterility that has allowed the establishment of diverse populations to perform recurrent selection on wheat (Triticum aestivum L.). Such is the case of the broad genetic background male sterile population called PANDOLY.PABG, which has allowed the development of lines and the generation of varieties. Eighty $F_{5}$ lines randomly taken from cycles $\mathrm{C} 0, \mathrm{C} 2, \mathrm{C} 4, \mathrm{C} 6, \mathrm{C} 8, \mathrm{C} 10, \mathrm{C} 12, \mathrm{C} 14$ and 10 control varieties were evaluated to estimate genetic advancement after 14 cycles of recurrent selection in the population mentioned above and to assess the efficiency of the Oly gene to form superior lines. A $9 \times 10$ alpha-lattice design was used with two replications and two planting dates during the Autumn-Winter 2017/2018 cycle in Roque, Guanajuato, and Montecillo, State of Mexico. The variables evaluated were days to flowering $(\mathrm{DF})$, plant height $(\mathrm{PH})$, days to maturity (DM), grain yield (GY), grain filling rate (GFR), harvest index (HI) biomass (BIO), weight of 1000 seeds (WTS), spikes per $\mathrm{m}^{2}$ (EPM2), grains per $\mathrm{m}^{2}$ (GPM2) and grains per spike (GPE). The highest increase in GY was 1445 $\mathrm{kg} \mathrm{ha}^{-1}$ between cycles $\mathrm{C} 0$ and $\mathrm{C} 2$. The average genetic progress in the 14 cycles was $3.58 \%$ ( $140.6 \mathrm{~kg}$ ha-1/cycle). Among the morpho-physiological changes observed, particularly in advanced selection cycles, were an increase $(P \leq 0.05)$ in GFR, HI, BIO, WTS, EPM2, GPM2, and GPE, and a decrease (P $\leq$ $0.05)$ of DF, DM and PH. The control varieties and the lines derived from $\mathrm{C} 14$ presented higher $\mathrm{GY}(\mathrm{P} \leq 0.05)$ and were statistically equal to each other. The Oly gene is an excellent facilitator of population genetic improvement in wheat.

Index words: Triticum aestivum, male sterility, MSFRS technique, selection cycle.

\section{INTRODUCCIÓN}

La selección recurrente es un proceso cíclico de selección-recombinación entre individuos sobresalientes, diseñado para acumular combinaciones de genes favorables, mantener la variabilidad genética y obtener avances genéticos (Ramya et al., 2016), y tiene diversos beneficios tales como el rápido desarrollo de un ciclo de selección y la creación de genotipos superiores por la acumulación continua de alelos favorables, gracias a la facilidad de cruzamiento entre docenas de líneas progenitoras (Pang et al., 2017), lo cual permite la ruptura de bloques de ligamiento (Zhang et al., 2015).

La aplicación de la selección recurrente no es común en especies autógamas debido a la cantidad de mano de obra requerida para cruzar los genotipos seleccionados (Zhang et al., 2015). En el caso del trigo, una alternativa para facilitar el cruzamiento de líneas es la inducción de la androesterilidad, para lo cual se reporta el uso de: i) agentes o productos químicos, ii) androesterilidad citoplasmática basada en reordenamientos del ADN mitocondrial, iii) androesterilidad nuclear producto de mutaciones en genes codificados en el núcleo (Whitford et al., 2013); 
esta última acrecienta la posibilidad de seleccionar los mejores genotipos a lo largo de la selección recurrente porque mantiene la variabilidad en la población y no hay efectos negativos en el rendimiento, puesto que se logra la restauración completa de la fertilidad (Whitford et al., 2013). Existen genes que causan androesterilidad en trigo como ms 1 y ms 5 que son recesivos, y ms2, ms3 y ms 4 que son dominantes (Singh et al., 2015).

La androesterilidad en la selección recurrente es usada como una herramienta para realizar mejoramiento poblacional; Ramage (1977) denominó a esta técnica "Male Sterility Facilitated Recurrent Selection" (MSFRS). Ramya et al. (2016) realizaron cruzas masivas en $\mathrm{F}_{5}$ a partir de una cruza simple en trigo (HI $1500 \times$ HUW 510) y obtuvieron ganancias para rendimiento de grano de $17 \%$. En México, Solís-Moya et al. (2002) y Villaseñor et al. (2002), para la misma variable, reportaron avances genéticos de 1.9 y 4.7 $\%$ por ciclo de MSFRS en trigo, respectivamente.

Villaseñor et al. (2014) reportaron el hallazgo de un gen nuclear dominante que induce androesterilidad en trigo, al cual denominaron Oly; no está ligado con efectos deletéreos, por lo tanto, es empleado para facilitar la selección recurrente. El gen Oly incorporado a través de retrocruzas a genotipos elite ha permitido conformar diversas poblaciones (Villaseñor et al., 2015); una de ellas es la denominada población androestéril dominante Oly de amplia base genética (PANDOLY.PABG) (Villaseñor et al., 2014; Villaseñor et al., 2015). El objetivo de este trabajo fue estimar el avance genético después de 14 ciclos de selección recurrente en la población androestéril PANDOLY. PABG y valorar sus ventajas para facilitar la formación de líneas superiores fértiles con características morfofisiológicas favorables.

\section{MATERIALES Y MÉTODOS}

\section{Generación de la población}

La población PANDOLY.PABG fue formada en el programa de mejoramiento genético de trigo de temporal del Campo Experimental Valle de México (CEVAMEX) perteneciente al Instituto Nacional de Investigaciones Forestales, Agrícolas y Pecuarias (INIFAP); se utilizaron 20 progenitores del ciclo primavera-verano 2003, mismos que se cruzaron con plantas androestériles portadoras de gen Oly; de esta recombinación, a plantas androestériles $F_{1}$ se les realizaron dos retrocruzas con los progenitores para incorporarles a éstos el carácter de androesterilidad. En el ciclo primavera-verano 2005 semilla de los progenitores androestériles se utilizó para formar un compuesto balanceado que se sometió a un ciclo de apareamiento aleatorio; la semilla recombinante de las plantas androestériles se utilizó para obtener la población original (C0). La semilla del C0 se sembró para realizar el primer ciclo de selección recurrente (C1); la semilla de las plantas androestériles seleccionadas visualmente por tipo agronómico y reacción a enfermedades se utilizó para el C2 y la semilla de las plantas fértiles seleccionadas se utilizó para representar a su ciclo origen (C0); este procedimiento se realizó sucesivamente hasta llegar al C14. En cada ciclo el tamaño de la población fue de 10,000 plantas; se realizó selección masal con control de las hembras; durante la floración se seleccionó el 5 \% de las plantas androestériles, que se dejaron a libre apareamiento; esas plantas se cosecharon masivamente, generando su respectivo ciclo de selección recurrente (Villaseñor et al., 2018).

\section{Avance generacional $F_{3}-F_{5}$}

Se utilizó semilla remanente cosechada de plantas fértiles $\left(F_{2}\right)$ de ocho ciclos (C0, C2, C4, C6, C8, C10, C12 y C14) donde se aplicó la técnica MSFRS. Durante el ciclo otoño-invierno 2016-2017 se marcaron 100 plantas fértiles $\mathrm{F}_{3}$ de cada ciclo y se cosecharon individualmente; éstas se incrementaron durante el ciclo primavera-verano 2017 y se cosecharon al azar 50 familias por ciclo; la semilla $\mathrm{F}_{5}$ de 10 familias se tomaron al azar de cada ciclo en estudio y se utilizaron para la evaluación del avance genético.

\section{Ensayo de rendimiento}

El ensayo de rendimiento se conformó por 80 familias (10 familias por ciclo de selección) más 10 variedades como testigos (Rebeca F2000, Tlaxcala F2000, Náhuatl F2000, Tacupeto F2001, Bárcenas S2002, Urbina S2007, Nana F2007, Norteña F2007, Bourlaug 100 y Conatrigo F2015). Para la evaluación se utilizó un diseño alfa-látice $9 \times 10$ con dos repeticiones, en dos localidades y dos fechas de siembra en cada una, el 5 y 15 de diciembre en Roque, Celaya, Guanajuato, y el 20 de diciembre y 19 de enero en Montecillo, Texcoco, Estado de México durante el ciclo otoño-invierno 2017-2018. En ambos sitios se aplicaron cinco riegos, a los 0, 35, 65, 85 y 105 días después de la siembra. La unidad experimental (UE) consistió en cuatro surcos de $1 \mathrm{~m}$ de longitud, con distancia entre surcos de $0.30 \mathrm{~m}$ y una densidad de siembra de $120 \mathrm{~kg} \mathrm{ha}^{-1}$. Se empleó la dosis de fertilización $160 \mathrm{~N}-80 \mathrm{P}-00 \mathrm{~K}$, la cual se aplicó al voleo en la siembra. Las malezas se controlaron oportunamente con Topik ${ }^{\circledR}$ 240EC (700 mL ha-1) y Esteron ${ }^{\circledR} 47 \mathrm{M}\left(750 \mathrm{~mL} \mathrm{ha}^{-1}\right)$; también se aplicó Folicur ${ }^{\circledR}\left(500 \mathrm{~mL} \mathrm{ha}^{-1}\right)$ para el control de roya lineal (Puccinia striiformis f. sp. tritici W.). 


\section{Variables evaluadas}

Los caracteres que se registraron fueron días a floración (DF), desde la siembra hasta que el $50 \%$ de las espigas estaban expuestas; la altura de planta (AP, cm) se midió a la madurez fisiológica del grano desde la superficie del suelo hasta el ápice de la espiga; los días a madurez (DM), contados desde la siembra hasta que $50 \%$ de los pedúnculos de las espigas se tornó amarillento; rendimiento de grano ( $\left.R G, \mathrm{~kg} \mathrm{ha}^{-1}\right)$ se estimó a partir del peso del grano cosechados por UE; tasa de llenado de grano (TLLG, $\mathrm{kg} \mathrm{d}^{-1}$ ) $=R G / P L L G$, donde el periodo de llenado de grano (PLLG) = DM - DF; índice de cosecha $(\mathrm{IC})=\mathrm{RE} / \mathrm{RB}$, donde RE es el rendimiento de granos en $\mathrm{g}$ de 20 tallos por UE y RB el rendimiento biológico aéreo en $\mathrm{g}$ de 20 tallos por $\mathrm{UE}$; biomasa aérea $\left(\mathrm{BIO}, \mathrm{g} \mathrm{m}^{-2}\right)=[(\mathrm{RC}+\mathrm{RE}) / \mathrm{UE}] / \mathrm{IC}$, donde $\mathrm{RC}$ es el rendimiento de grano por unidad experimental (g), UE es la superficie de la parcela experimental en $\mathrm{m}^{2}$; peso de 1000 semillas (PMS, g), producto del peso de 200 semillas multiplicado por cinco; espigas por $\mathrm{m}^{2}(\mathrm{EPM} 2)=(\mathrm{BIO} / \mathrm{RB})$ $\times 20$; granos por $\mathrm{m}^{2}(\mathrm{GPM} 2)=[(\mathrm{RC}+\mathrm{RE}) / \mathrm{UE}] /[(\mathrm{PMS} / 1000)]$; y granos por espiga (GPE), mediante el cociente GPM2/ EPM2 (Solís-Moya et al., 2002).

\section{Análisis estadístico}

Se realizó análisis de varianza combinado con el paquete estadístico SAS 9.4 (SAS Institute, 2013), en el cual los factores de variación fueron los ambientes (combinación entre localidades y fechas de siembra), ciclos de selección, familias e interacción ambientes $\times$ ciclos. Se realizó comparación de medias con la prueba de Tukey con una probabilidad de $\mathrm{P} \leq$ 0.05. El avance genético ( $A G)$ se calculó con la fórmula: $A G$ $=\left(b i / X_{c 0}\right) \times 100$ (donde bi es el coeficiente de regresión lineal de la media de cada carácter a través de ciclos y $\mathrm{X}_{\mathrm{co}}$ es el promedio de cada carácter en el C0) (Benítez, 2001).
Se obtuvo una regresión lineal para las variables $R G$ GPM2, GPE, TLLG e IC con las medias de cada ciclo (CO al C14); además, se compararon con la media de testigos (MT) y con la media del cultivar Nana F2007, debido a que la población PANDOLY.PABG a lo largo de los 14 ciclos de selección sólo ha derivado variedades para secano, como Valles F2015 (Villaseñor et al., 2018).

\section{RESULTADOS Y DISCUSIÓN}

En el análisis combinado se detectaron diferencias significativas entre ambientes, ciclos y familias para todas las variables; la interacción ambientes $\times$ ciclos $(A \times C)$ fue significativa para la mayoría de los caracteres, excepto para IC y AP (Cuadros 1 y 2). Los ambientes no tuvieron un efecto significativo sobre la relación grano-rastrojo de los genotipos, lo que se explica porque la diferencia entre los ambientes radica en fechas de siembra y localidades, y no por algún tipo de estrés inducido.

Para los caracteres que describen la fenología, la comparación de medias mostró que el C0y C2 son los ciclos que requirieron más días para llegar a floración, mostrando diferencia con el resto de los ciclos, mientras que las variedades testigo (VT) y el C12 fueron los que registraron menos días para esa variable (Cuadro 3). Para DM los C0 y C14 no presentaron diferencias entre ellos, pero sí cuando se compararon con las VT, C12 y C4, lo que significa que la selección para este carácter no presentó una relación lineal a lo largo de los ciclos de selección recurrente, pues no es la principal característica considerada durante la selección en el programa de mejoramiento. La selección redujo la altura de planta a un porte similar al de los testigos. En cuanto a la TLLG, se observó que las VT presentaron diferencia estadística con el C0 y similitud con el C14. El IC y la BIO se incrementaron con el avance a través de los

Cuadro 1 Cuadrados medios del análisis de varianza para días a floración (DF), madurez fisiológica (DM), altura de planta (AP), tasa de llenado de grano (TLLG), índice de cosecha (IC) y biomasa aérea (BIO).

\begin{tabular}{|c|c|c|c|c|c|c|c|}
\hline $\mathrm{FV}$ & $\mathrm{GL}$ & DF & $\mathrm{DM}$ & $\mathrm{AP}$ & TLLG & $\mathrm{IC}$ & $\mathrm{BIO}$ \\
\hline A & 3 & $17,127.2$ ** & $19,986.5 * *$ & $7688.1 * *$ & $363,425.3 * \star$ & $0.0701 * *$ & $69,587,248 * *$ \\
\hline $\mathrm{R}(\mathrm{A})$ & 4 & $30.2 * *$ & $24.3 * *$ & $115.3 *$ & $15759.2 * *$ & $0.0132 * *$ & $25,83,186 * *$ \\
\hline$S B(A \times R)$ & 72 & 3.5 & 4.1 & 36.6906 & 331.8 & 0.0011 & 99,474 \\
\hline C & 8 & $209.8^{* *}$ & $133.9 \star \star$ & $3868.4 * \star$ & $14941.0 * \star$ & $0.0705 * *$ & $706,485 * *$ \\
\hline$A \times C$ & 24 & $12.7 \star \star$ & $22.4 \star \star$ & 53.5 & $1254.7 * \star$ & 0.0016 & $139,018 * *$ \\
\hline F & 81 & $93.6 * \star$ & $107.3 * \star$ & $1285.2^{\star \star *}$ & $2572.1 * *$ & $0.0111 * *$ & $233,559 * *$ \\
\hline Error & 527 & 4.4 & 5.5 & 35.5 & 426.5 & 0.0013 & $38,468,077$ \\
\hline$R^{2}$ & & 0.96 & 0.96 & 0.90 & 0.87 & 0.73 & 0.86 \\
\hline CV (\%) & & 2 & 2 & 6 & 19 & 9 & 20 \\
\hline
\end{tabular}

FV: fuentes de variación, GL: grados de libertad, A: ambientes, R: repeticiones, SB: sub-bloques, C: ciclos, F: familias, R²: coeficiente de determinación, $C V$ : coeficiente de variación, *: significancia con $\mathrm{P} \leq 0.05, * *$ : significancia con $\mathrm{P} \leq 0.01$. 
Cuadro 2. Cuadrados medios del análisis de varianza para rendimiento de grano (RG) y los componentes del rendimiento peso de mil semillas (PMS), espigas por $\mathrm{m}^{2}$ (EPM2), granos por $\mathrm{m}^{2}$ (GPM2) y granos por espiga (GPE).

\begin{tabular}{|c|c|c|c|c|c|c|}
\hline $\mathrm{FV}$ & $\mathrm{GL}$ & $R G$ & PMS & EPM2 & GPM2 & GPE \\
\hline A & 3 & $387,689,918 * *$ & $1235.4 * \star$ & $6,083,737 \star \star$ & $11,412,951,494 * *$ & $2608.6 * *$ \\
\hline$R(A)$ & 4 & $30,486,724 \star \star$ & 10.5 & $198,108 * *$ & $392,656,361 * *$ & $393.3 * *$ \\
\hline$S B(A \times R)$ & 72 & 928,214 & 7.6 & 11,738 & $12,289,711$ & 77.9 \\
\hline C & 8 & $49,477,867 \star \star *$ & $229.5 * \star$ & $19,709 *$ & $513,659,435 * \star$ & $2477.5 * \star$ \\
\hline$A \times C$ & 24 & $2,320,745 * *$ & $10.6 *$ & 13,165 & $41,969,499 * *$ & 56.5 \\
\hline F & 81 & $7,711,064 \star *$ & $107.2 * *$ & $21,529 * *$ & $112,603,668 * *$ & 440.1 ** \\
\hline Error & 527 & $1,016,836$ & 6.7 & $4,803,945$ & $15,860,348$ & 41.1 \\
\hline$R^{2}$ & & 0.82 & 0.81 & 0.82 & 0.86 & 0.63 \\
\hline CV (\%) & & 18 & 6 & 25 & 21 & 17 \\
\hline
\end{tabular}

FV: fuentes de variación, GL: grados de libertad, A: ambientes, R: repeticiones, SB: sub-bloques, C: ciclos, F: familias, R²: coeficiente de determinación, CV: coeficiente de variación, *: significancia con $\mathrm{P} \leq 0.05, * *$ : significancia con $\mathrm{P} \leq 0.01$.

ciclos de selección hasta valores estadísticamente iguales al promedio de los testigos. Los resultados en BIO son opuestos a los obtenidos por Sánchez-García et al. (2013), quienes reportaron que la selección no incrementó esta variable.

La selección a través de los ciclos tuvo un efecto positivo sobre el rendimiento, ya que logró un incremento de 2288 $\mathrm{kg} \mathrm{ha}^{-1}$; sin embargo, el rendimiento del mejor ciclo (C14) sólo igualó el rendimiento promedio de los testigos (Cuadro 4). En relación con los componentes de rendimiento, en el carácter PMS, el C12 tuvo la media más alta, incluso por encima de las $V T$, y fue estadísticamente diferente del resto. Los C14 y C0 no presentaron diferencias significativas para PMS, lo que refleja ausencia de selección para esta variable. Para GPM2 el C14 presentó el mayor número de granos seguido por las VT y el C10. El incremento en el rendimiento en este estudio se relacionó con el aumento en GPM2 y GPE ( $r=0.98 * * ; r=0.96 * *)$ (Gaju et al., 2009; Molero et al., 2016); el C0 presentó la menor cantidad de espigas y fue estadísticamente diferente de las VT y C14; además, hubo un incremento sustancial de GPE a lo largo de los ciclos.

El mayor aumento de RG fue de $1445 \mathrm{~kg} \mathrm{ha}^{-1}$ y se presentó entre los C0 y C2; en el C4 RG disminuyó, pero a partir del C6 y hasta el C14 éste incrementó (Cuadro 4); resultados similares reportaron Solís-Moya et al. (2002). El avance genético logrado después de 14 ciclos en las variables DF, DM y AP fue negativo, lo que significa que hubo reducción de las variables a través del proceso de selección recurrente, debido a que la selección se dirigió hacia ciclo precoz y porte bajo de planta. La precocidad es un carácter que se relaciona con el incremento de

Cuadro 3. Comparación de medias entre ciclos y variedades testigo (VT) de los caracteres morfofisiológicos.

\begin{tabular}{lllcccc}
\hline Ciclo & DF $(\mathrm{d})$ & DM $(\mathrm{d})$ & AP $(\mathrm{cm})$ & TLLG $\left(\mathrm{kg} \mathrm{d}^{-1}\right)$ & IC & BIO $\left(\mathrm{g} \mathrm{m}^{-2}\right)$ \\
\hline C0 & $85.1 \mathrm{a}$ & $130.4 \mathrm{a}$ & $115.3 \mathrm{a}$ & $76.7 \mathrm{e}$ & $0.33 \mathrm{e}$ & $1143.1 \mathrm{c}$ \\
$\mathrm{C} 2$ & $84.5 \mathrm{a}$ & $130.2 \mathrm{ab}$ & $107.0 \mathrm{~b}$ & $103.8 \mathrm{~cd}$ & $0.38 \mathrm{~cd}$ & $1310.9 \mathrm{c}$ \\
$\mathrm{C} 4$ & $81.1 \mathrm{~cd}$ & $126.9 \mathrm{e}$ & $108.5 \mathrm{~b}$ & $97.7 \mathrm{~d}$ & $0.37 \mathrm{~d}$ & $1282.98 \mathrm{~b}$ \\
$\mathrm{C6}$ & $82.7 \mathrm{~b}$ & $129.0 \mathrm{bc}$ & $97.6 \mathrm{~d}$ & $105.7 \mathrm{~cd}$ & $0.40 \mathrm{bc}$ & $1298.15 \mathrm{~b}$ \\
C8 & $81.4 \mathrm{c}$ & $128.4 \mathrm{~cd}$ & $103.4 \mathrm{c}$ & $105.9 \mathrm{~cd}$ & $0.39 \mathrm{c}$ & $1341.7 \mathrm{bc}$ \\
C10 & $81.8 \mathrm{bc}$ & $129.6 \mathrm{ab}$ & $93.0 \mathrm{e}$ & $111.8 \mathrm{bc}$ & $0.42 \mathrm{a}$ & $1347.7 \mathrm{abc}$ \\
C12 & $80.4 \mathrm{de}$ & $127.0 \mathrm{e}$ & $98.9 \mathrm{~d}$ & $112.0 \mathrm{bc}$ & $0.41 \mathrm{ab}$ & $1358.8 \mathrm{abc}$ \\
C14 & $82.6 \mathrm{~b}$ & $130.3 \mathrm{a}$ & $97.9 \mathrm{~d}$ & $121.7 \mathrm{ab}$ & $0.41 \mathrm{ab}$ & $1475.5 \mathrm{a}$ \\
VT & $80.1 \mathrm{e}$ & $127.8 \mathrm{de}$ & $96.2 \mathrm{~d}$ & $122.7 \mathrm{a}$ & $0.43 \mathrm{a}$ & $1460.1 \mathrm{a}$ \\
DSH (0.05) & 1.03 & 1.16 & 2.93 & 10.17 & 0.017 & 133.28 \\
\hline
\end{tabular}

Medias con la misma letra en las columnas son estadísticamente iguales (Tukey, P $\leq 0.05)$. DF: días a floración, DM: días a madurez fisiológica, AP. altura de planta, PLLG: periodo de llenado de grano, TLLG: tasa de llenado de grano, IC: índice de cosecha, BIO: biomasa, DSH: diferencia significativa honesta. 
Cuadro 4. Comparación de medias entre ciclos y variedades testigo (VT) del rendimiento y sus componentes.

\begin{tabular}{lccccc}
\hline Ciclo & RG $\left(\mathrm{kg} \mathrm{ha}^{-1}\right)$ & PMS (g) & EPM2 & GPM2 & GPE \\
\hline C0 & $3929.8 \mathrm{f}$ & $40.5 \mathrm{c}$ & $345.2 \mathrm{~b}$ & $13,166.6 \mathrm{e}$ & $39.3 \mathrm{~d}$ \\
C2 & $5375.6 \mathrm{de}$ & $39.9 \mathrm{c}$ & $374.2 \mathrm{ab}$ & $17,911.7 \mathrm{~cd}$ & $49.7 \mathrm{bc}$ \\
C4 & $5040.1 \mathrm{e}$ & $42.5 \mathrm{~b}$ & $370.3 \mathrm{ab}$ & $16,386.5 \mathrm{~d}$ & $45.5 \mathrm{c}$ \\
C6 & $5514.1 \mathrm{cde}$ & $38.5 \mathrm{~d}$ & $376.2 \mathrm{ab}$ & $19,340.3 \mathrm{bc}$ & $52.7 \mathrm{ab}$ \\
C8 & $5542.4 \mathrm{~cd}$ & $39.4 \mathrm{~cd}$ & $384.5 \mathrm{ab}$ & $19,229.9 \mathrm{bc}$ & $53.2 \mathrm{ab}$ \\
C10 & $6064.7 \mathrm{ab}$ & $39.5 \mathrm{~cd}$ & $379.7 \mathrm{ab}$ & $20,334.1 \mathrm{ab}$ & $56.2 \mathrm{a}$ \\
C12 & $5930.4 \mathrm{cb}$ & $44.1 \mathrm{a}$ & $351.6 \mathrm{ab}$ & $17,833.2 \mathrm{~cd}$ & $53.6 \mathrm{ab}$ \\
C14 & $6465.9 \mathrm{a}$ & $40.6 \mathrm{c}$ & $393.8 \mathrm{a}$ & $21,359.6 \mathrm{a}$ & $56.4 \mathrm{a}$ \\
VT & $6524.1 \mathrm{a}$ & $42.8 \mathrm{~b}$ & $396.89 \mathrm{a}$ & $20,798.5 \mathrm{ab}$ & $54.1 \mathrm{ab}$ \\
DSH (0.05) & 496.6 & 1.27 & 47.09 & 1961.3 & 8.5 \\
\hline
\end{tabular}

Medias con la misma letra en las columnas son estadísticamente iguales (Tukey, $\mathrm{P} \leq 0.05)$. RG: rendimiento de grano, PMS: peso de mil semillas, EPM2: espigas por m², GPM2: granos por m², GPE: granos por espiga, NEE: número de espiguillas por espiga, LDE: Iongitud de espiga, DSH: diferencia significativa honesta.

rendimiento de genotipos en el noroeste de México; así mismo, la altura de planta no se relaciona con esta variable, pues la mayor parte de los materiales utilizados posee genes Rht (Lopes et al., 2012). El avance genético en el resto de las variables fue positivo, lo que indica que hay un incremento en su expresión (Cuadro 5). El RG tuvo el mayor incremento por ciclo $\left(3.58 \%, 140.6 \mathrm{~kg} \mathrm{ha}^{-1}\right)$; es decir, $7.16 \%$ anual, porcentaje superior a lo reportado por Paquini-Rodríguez et al. (2016) en México y por Balota et al. (2017) en Estados Unidos de América, con ganancias anuales de $1.21 \%$ (41.77 $\left.\mathrm{kg} \mathrm{ha}^{-1}\right)$ y $1.11 \%\left(46.7 \mathrm{~kg} \mathrm{ha}^{-1}\right)$, respectivamente.

A nivel global Crespo-Herrera et al. (2017) midieron las ganancias en mega ambientes bajo condiciones óptimas de riego y reportaron aumentos de $0.72 \%\left(46.65 \mathrm{~kg} \mathrm{ha}^{-1}\right)$. Lo anterior indica que la MSFRS es un excelente método de mejoramiento genético poblacional en trigo, ya que se logra mayor efectividad en ganancia de rendimiento de grano y compite con los métodos tradicionales de fitomejoramiento; además, permite el apareamiento libre entre genotipos seleccionados en cada ciclo de selección, el proceso de recombinación es natural y como resultado se mantiene la variabilidad genética en la población.

De acuerdo con el avance genético obtenido (Cuadro 5), las variables fisiológicas y componentes de rendimiento más fuertemente relacionados con el aumento del rendimiento fueron TLLG $(r=0.99 * *)$, IC $(r=0.96 * *)$, GPE $(r$ $=0.97 * *)$ y GPM2 $(r=0.94 * *)$, resultados que coinciden con los de otros estudios (Beche et al., 2014; Sánchez-García et al., 2013; Sayre et al., 1997). El PMS no se encuentra correlacionado con el aumento en rendimiento $(r=0.008)$, contrario a lo que indicaron Murphy et al. (2008) y Lopes et al. (2012); por otro lado, Lopes y Reynolds (2012) indicaron que bajo condiciones de estrés de calor se presenta una relación negativa. El resultado de este estudio para esta variable se explica porque el PMS inicial (CO) fue alto y se mantuvo a través de los ciclos.

De acuerdo con la información mostrada en la Figura 1, para RG y TLLG la media del C14 no superó a MT y Nana F2007 (Figura 1); por otro lado, en los componentes de rendimiento GPM2 y GPE la población del C14 fue superior (Figura 1); estos resultados indican que se ha logrado un avance en dichas variables, pero no ha sido suficiente para superar el rendimiento de los testigos; sin embargo, gracias a la alta correlación que presentan dichas variables, se infiere que se pueden obtener mejores resultados en ciclos posteriores de selección recurrente.

La frecuencia de líneas superiores (LS) en MT y Nana F2007 se observó en mayor número en los ciclos avanzados (Cuadro 6). Para RG hubo ocho LS al mejor testigo de temporal, de las cuales el número 3 correspondió al C14; si se suman las LS de los C10, C12 y C14 se observa que hay mayor frecuencia en ciclos avanzados, lo anterior coincide con lo reportado por Solís-Moya et al. (2002) y Villaseñor et al. (2002). Para EPM2, de las 30 LS que superaron a Nana F2007, el 20 \% correspondió al C14. Para GPE se identificaron LS de los C6, C8, C10, C12 y C14. Es importante identificar este tipo de LS, porque de éstas se derivan líneas que darán origen a las futuras variedades, como es el caso de Valles F2015, que se originó del C4 de la población en estudio (Villaseñor et al., 2018). 
Cuadro 5. Avance genético por ciclo $(\Delta G / C)$ y anual ( $\Delta G /$ año) para caracteres fisiológicos y componentes de rendimiento en trigo después de 14 ciclos de selección recurrente.

\begin{tabular}{|c|c|c|c|c|c|}
\hline Carácter & $\mathrm{CO}$ & bi & $\mathrm{R}^{2}$ & $\Delta G / C$ & $\Delta G / a n ̃ o$ \\
\hline$D F(d)$ & 85.1 & -0.22 & 0.4368 & -0.26 & -0.52 \\
\hline $\mathrm{DM}(\mathrm{d})$ & 130.4 & -0.05 & 0.0354 & -0.04 & -0.08 \\
\hline $\mathrm{AP}(\mathrm{cm})$ & 115.3 & $-1.21 *$ & 0.6648 & -1.05 & -2.10 \\
\hline TLLG $\left(\mathrm{kg} \mathrm{d}^{-1}\right)$ & 76.7 & $2.37 * *$ & 0.7696 & 3.09 & 6.18 \\
\hline IC & 0.33 & $0.0051 * *$ & 0.7305 & 1.54 & 3.09 \\
\hline $\mathrm{BIO}\left(\mathrm{g} \mathrm{m}^{-2}\right)$ & 1143.1 & $16.69 * \star$ & 0.7781 & 1.46 & 2.92 \\
\hline$R G\left(\mathrm{~kg} \mathrm{ha}^{-1}\right)$ & 3929.8 & $140.64 * \star$ & 0.8048 & 3.58 & 7.16 \\
\hline PMS (g) & 40.5 & 0.077 & 0.0421 & 0.19 & 0.38 \\
\hline EPM2 & 345.2 & 1.56 & 0.2237 & 0.45 & 0.90 \\
\hline GPM2 (d) & $13,166.6$ & $408.87 *$ & 0.614 & 3.11 & 6.21 \\
\hline GPE & 39.3 & $1.02 \star *$ & 0.7342 & 2.60 & 5.19 \\
\hline
\end{tabular}

CO: medias del ciclo original, bi: coeficiente de regresión lineal de la media de cada variable sobre el número de ciclos de selección, $\mathrm{R}^{2}$ : coeficiente de determinación, DF: días a floración, DM: días a madurez fisiológica, AP. altura de planta, TLLG: tasa de llenado de grano, IC: índice de cosecha, BIO: biomasa, RG: rendimiento de grano, PMS: peso de mil semillas, EPM2: espigas por $\mathrm{m}^{2}$, GPM2: granos por m², GPE: granos por espiga, *: significativo con $\mathrm{P} \leq 0.05, * *$ : significativo con $\mathrm{P} \leq 0.01$.

\section{CONCLUSIONES}

Después de 14 ciclos de selección recurrente en la población PONDOLY.PABG se incrementó el rendimiento de grano en $3.58 \%$ (140.6 $\mathrm{kg} \mathrm{ha}^{-1}$ ) por ciclo, con un total acumulado en siete años (dos ciclos por año) de $50.1 \%$ (1968.9 $\left.\mathrm{kg} \mathrm{ha}^{-1}\right)$, aunque la selección sólo logró superar la depresión del rendimiento generada al formar la población, ya que el ciclo C14 apenas igualó el rendimiento de la media de los testigos. Los componentes de rendimiento donde hubo mayor respuesta a la selección recurrente con el uso de la técnica MSFRS fueron granos por espiga y granos por $\mathrm{m}^{2}$, mientras que los componentes fisiológicos fueron índice de cosecha y tasa de llenado de grano. La selección recurrente facilitada por la androesterilidad es una técnica práctica de mejoramiento genético poblacional en trigo que permite mayor avance genético, facilita la recombinación entre los individuos, compite con los métodos tradicionales de fitomejoramiento y es efectiva para generar líneas superiores.

\section{BIBLIOGRAFÍA}

Balota M., A. J. Green, C. A. Griffey, R. Pitman and W. Thomason (2017) Genetic gains for physiological traits associated with yield in soft red winter wheat in the Eastern United States from 1919 to 2009. European Journal of Agronomy 84: 76-83, https://doi.org/10.1016/j.eja.2016.11.008

Beche E., G. Benin, C. L. da Silva, L. B. Munaro and J. A. Marchese (2014) Genetic gain in yield and changes associated with physiological traits in Brazilian wheat during the 20th century. European Journal of Agronomy 61:49-59, https://doi.org/10.1016/j.eja.2014.08.005

Benítez R. I. (2001) Comportamiento de líneas $\mathrm{S}_{3}$ de trigo derivadas de poblaciones mejoradas por selección recurrente. Revista
Fitotecnia Mexicana 24:63-70.

Crespo-Herrera L. A., J. Crossa, J. Huerta-Espino, E. Autrique, S. Mondal, G. Velu, ... and R. P. Singh (2017) Genetic yield gains in CIMMYT's international elite spring wheat yield trials by modeling the genotype $\times$ environment interaction. Crop Science 57:789-801, https://doi.org/10.2135/cropsci2016.06.0553

Gaju O., M. P. Reynolds, D. L. Sparkes and M. J. Foulkes (2009) Relationships between large-spike phenotype, grain number, and yield potential in spring wheat. Crop Science 49:961-973, https://doi.org/10.2135/cropsci2008.05.0285

Lopes M. S. and M. P. Reynolds (2012) Stay-green in spring wheat can be determined by spectral reflectance measurements (normalized difference vegetation index) independently from phenology. Journal of Experimental Botany 63:3789-3798, https://doi.org/10.1093/jxb/ers071

Lopes M. S., M. P. Reynolds, Y. Manes, R. P. Singh, J. Crossa and H. J. Braun (2012) Genetic yield gains and changes in associated traits of CIMMYT spring bread wheat in a "historic" set representing 30 years of breeding. Crop Science 52:11231131, https://doi.org/10.2135/cropsci2011.09.0467

Molero G., G. A. Slafer, A. C. Rivera-Amado, F. J. Pinera-Chavez, E. TrujilloNegrellos, A. Quintero, ... and M. P. Reynolds (2016) Traits determining differences in yield potential among elite lines of a spring wheat panel with the view to accelerating genetic gains. In: Proceedings of the 2nd International TRIGO (Wheat) Yield Potential Workshop 2016. M. Reynolds, G. Molero and E. Quilligan (eds.). Cd. Obregón, Sonora, Mexico, 9-10 March. CIMMYT. México, D. F. pp:25-34.

Murphy K. M., P. G. Reeves and S. S. Jones (2008) Relationship between yield and mineral nutrient concentrations in historical and modern spring wheat cultivars. Euphytica 163:381-390, https://doi.org/10.1007/s10681-008-9681-x

Pang Y., K. Chen, X. Wang, J. Xu, J. Ali and Z. Li (2017) Recurrent selection breeding by dominant male sterility for multiple abiotic stresses tolerant rice cultivars. Euphytica 213:268. https://doi.org/10.1007/s10681-017-2055-5

Paquini-Rodríguez S. L., I. Benítez-Riquelme, H. E. Villaseñor-Mir, A. MuñozOrozco y H. Vaquera-Huerta (2016) Incremento en el rendimiento y sus componentes bajo riego normal y restringido de variedades mexicanas de trigo. Revista Fitotecnia Mexicana 39:367-378 https://doi.org/10.35196/rfm.2016.4.367-378

Ramage R. T. (1977) Varietal improvement of wheat through male 

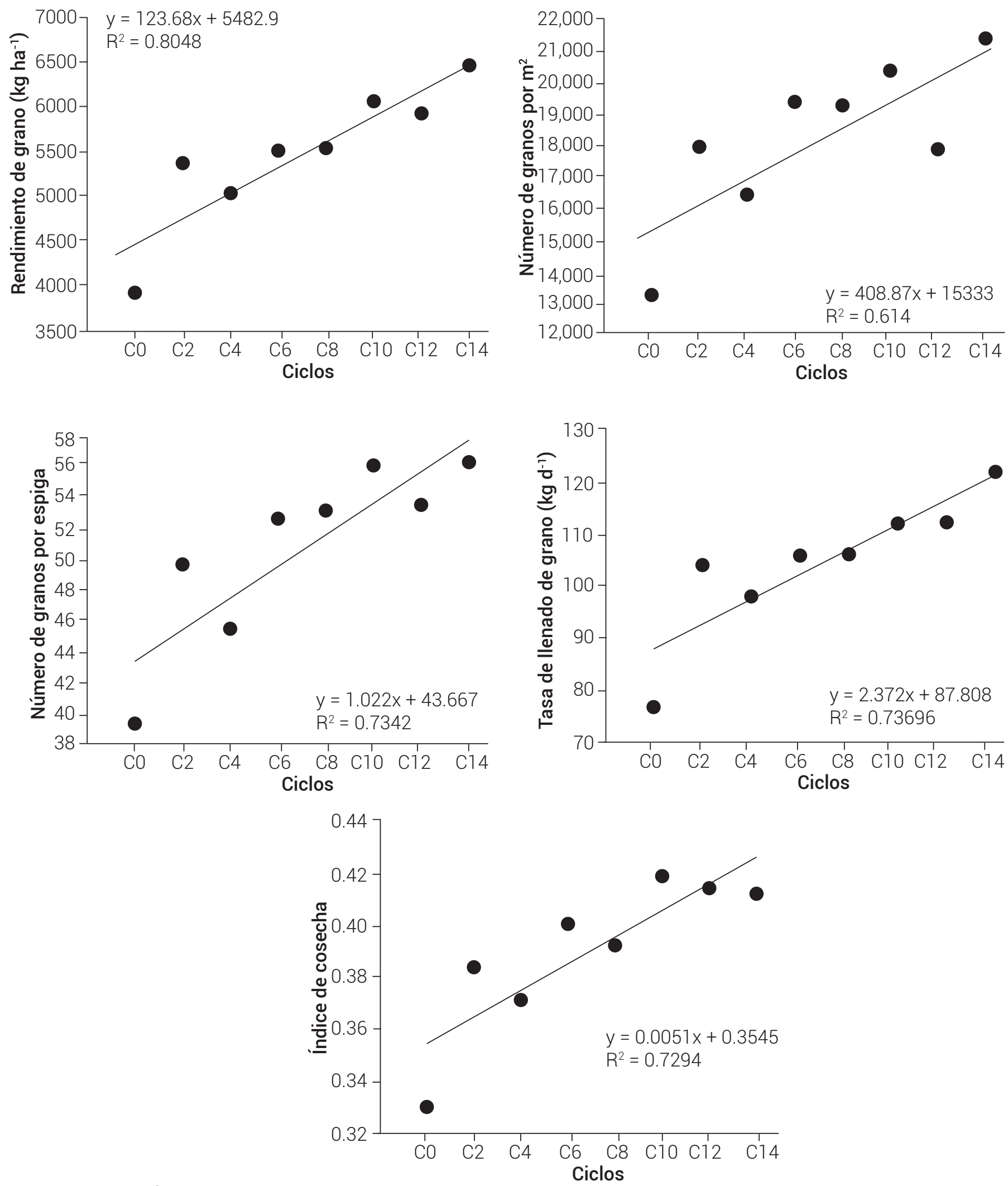

Figura 1. Evolución del rendimiento de grano, número de granos por $\mathrm{m}^{2}$, número de granos por espiga, tasa de llenado de grano e índice de cosecha a través de los ciclos de selección recurrente. 
Cuadro 6. Número de líneas superiores al testigo Nana F2007 y a la media de testigos para rendimiento de grano (RG) y componentes de rendimiento.

\begin{tabular}{|c|c|c|c|c|c|c|c|}
\hline & $R G$ & PMS & EPM2 & GPM2 & GPE & NEE & LDE \\
\hline & \multicolumn{7}{|c|}{ Líneas superiores a Nana F2007 } \\
\hline $\mathrm{CO}$ & 0 & 3 & 3 & 1 & 1 & 8 & 4 \\
\hline C2 & 2 & 1 & 4 & 4 & 4 & 10 & 2 \\
\hline C4 & 0 & 3 & 3 & 2 & 3 & 4 & 1 \\
\hline C6 & 1 & 1 & 3 & 5 & 6 & 7 & 1 \\
\hline C8 & 0 & 2 & 5 & 4 & 6 & 7 & 2 \\
\hline C10 & 1 & 2 & 5 & 6 & 8 & 5 & 3 \\
\hline C12 & 1 & 5 & 1 & 1 & 6 & 8 & 3 \\
\hline C14 & 3 & 0 & 6 & 5 & 6 & 9 & 2 \\
\hline \multirow[t]{2}{*}{$\mathrm{T}$} & 8 & 17 & 30 & 28 & 40 & 58 & 18 \\
\hline & \multicolumn{7}{|c|}{ Líneas superiores a la media de testigos } \\
\hline $\mathrm{CO}$ & 1 & 1 & 0 & 0 & 0 & 2 & 5 \\
\hline C2 & 0 & 0 & 0 & 0 & 0 & 3 & 5 \\
\hline C4 & 1 & 1 & 1 & 0 & 0 & 1 & 3 \\
\hline C6 & 1 & 0 & 0 & 0 & 0 & 1 & 6 \\
\hline C8 & 0 & 1 & 0 & 0 & 0 & 1 & 3 \\
\hline C10 & 2 & 1 & 0 & 1 & 0 & 1 & 2 \\
\hline C12 & 0 & 4 & 1 & 0 & 0 & 2 & 4 \\
\hline C14 & 1 & 1 & 0 & 0 & 0 & 1 & 5 \\
\hline $\mathrm{T}$ & 6 & 9 & 2 & 1 & 0 & 12 & 33 \\
\hline
\end{tabular}

GPE: granos por espiga, NEE: número de espiguillas por espiga, LDE: longitud de espiga, PMS: peso de mil semillas, EPM2: espigas por metro cuadrado, GPM2: granos por metro cuadrado, T: total.

sterile facilitated recurrent selection. Technical Bulletin No. 37. Asian and Pacific Council. Food and Fertilizer Technology Center. Taipei. $6 \mathrm{p}$.

Ramya P., G. P. Singh, N. Jain, P. K. Singh, M. K. Pandey, K. Sharma, ... and K. V. Prabhu (2016) Effect of recurrent selection on drought tolerance and related morpho-physiological traits in bread wheat. PloS ONE 11(6):e0156869, https://doi.org/10.1371/journal.pone.0156869

Sánchez-García M., C. Royo, N. Aparicio, J. A. Martín-Sánchez and F. Álvaro (2013) Genetic improvement of bread wheat yield and associated traits in Spain during the 20th century. Journal of Agricultural Science 151:105-118, https://doi.org/10.1017/S0021859612000330

SAS Institute (2013) Base SAS ${ }^{\circledR} 9.4$ Procedures Guide. Seventh edition. SAS Institute Inc. Cary, North Carolina, USA. 2638 p.

Sayre K. D., S. Rajaram and R. A. Fischer (1997) Yield potential progress in short bread wheats in northwest Mexico. Crop Science 37:36-42, https://doi.org/10.2135/cropsci1997.0011183X003700010006x

Singh S. P., R. Srivastava and J. Kumar (2015) Male sterility systems in wheat and opportunities for hybrid wheat development. Acta Physiologiae Plantarum 37:1713, https://doi.org/10.1007/s11738-014-1713-7

Solís-Moya E., H. E. Villaseñor-Mir, J. D. Molina-Galán, E. Espitia-Rangel, T. Cervantes-Santana y A. Martínez-Garza (2002) Selección masal visual recurrente para rendimiento de grano en una población androestéril de trigo harinero. Agrociencia 36:191-200.

Villaseñor M. H. E., F. Castillo G., S. Rajaram, E. Espitia R. y J. D. Molina G. (2002) Selección recurrente para rendimiento de grano en una población androestéril de trigo. Agricultura Técnica en México 28:43-52.

Villaseñor M. H. E., J. Huerta E., E. Espitia R., R. Hortelano S. R., M. F. Rodríguez G. y E. Martínez C. (2014) Genética y estabilidad del mutante androestéril dominante de trigo "Oly". Revista Mexicana de Ciencias Agrícolas 8:1509-1515, https://doi.org/10.29312/remexca.v0i8.1110

Villaseñor M. H. E., R. Hortelano S. R., E. Martínez C., J. Huerta E., E. García L. y E. Espitia R. (2015) Uso de la androesterilidad genética masculina en la reconversión de genotipos para realizar selección recurrente en trigo. Revista Mexicana de Ciencias Agrícolas 11:2177-2182 https://doi.org/10.29312/remexca.v0i1 1.795

Villaseñor M. H., J. Huerta E., R. Hortelano S. R., E. Martínez C., E. Espitia R., E. Solís M., L. Osorio A. y M. Rodríguez G. (2018) Valles F2015: nueva variedad de trigo para siembras de temporal en México. Revista Mexicana de Ciencias Agrícolas 9:1315-1320, https://doi.org/10.29312/remexca.v9i6.1592

Whitford R., D. Fleury, J. C. Reif, M. Garcia, T. Okada, V. Korzun and P. Langridge (2013) Hybrid breeding in wheat: technologies to improve hybrid wheat seed production. Journal of Experimental Botany 64:5411-5428, https://doi.org/10.1093/jxb/ert333

Zhang L., R. A. Richards, A. G. Condon, D. C. Liu and G. J. Rebetzke (2015) Recurrent selection for wider seedling leaves increases early biomass and leaf area in wheat (Triticum aestivum L.). Journal of Experimental Botany 66:1215-1226, https://doi.org/10.1093/jxb/eru468 